\title{
Stress profile and thermal expansion of layered materials determined from surface stresses
}

\author{
Jürgen Malzbender ${ }^{\mathrm{a})}$ \\ Forschungszentrum Jülich GmbH, Institute for Materials and Processes in Energy Systems, \\ 52425 Jülich, Germany
}

(Received 11 February 2004; accepted 13 April 2004; published online 19 May 2004)

\begin{abstract}
A simple method of inferring the stress profile and the effective difference in thermal expansion or strain in an unconstrained elastic multilayer system from a measurement of a limited number of surface stresses as obtained for example using x-ray diffraction or Raman spectroscopy is outlined. Explicit relationships are given for bilayered systems. The analysis procedure is exemplified for literature data of electronics materials, solid oxide fuel cells and thermal barrier systems. Following the outlined procedure, a determination of the stress profile and difference in thermal expansion for composites of alternating layers is also possible. (c) 2004 American Institute of Physics.
\end{abstract}

[DOI: $10.1063 / 1.1759773$ ]

Layered materials find widespread use in electronic, magnetic, optical, and structural components. Such components can be subjected to residual stresses due to intrinsic processes, such as sintering or crystallization, mismatch in thermal expansion coefficient or mechanical loading. Theories to relate stress, strain and curvature to the mechanical and thermal loading have been established for isotropic materials, bilayered, ${ }^{1}$ multilayered composites ${ }^{2}$ and materials with property gradients. ${ }^{3}$ These theories can be used to determine the difference in strain or thermal expansion as well as the residual stresses in a layered composite from the change in deflection or curvature.

Residual stresses in the surface of coated and layered materials are often assessed using Raman spectroscopy or x-ray diffraction. ${ }^{4,5}$ However, a determination of the stress profile requires a large number of measurements. In the current letter it is shown how the stress profile can be determined from a limited number of measurements. Only the more common cases of bilayer (coating on a substrate) and trilayer composites are analyzed in detail. However, following the outlined procedure the stress profile and thermal expansion for composites of alternating layers can be determined.

It is assumed that the stress is measured in the surface of layer 1 . It has to be considered that the stress is only representative of the surface if the analyzed depth is small compared to the layer thickness or the stress is relatively constant over the layer thickness, which is the case if the film thickness is much smaller than the substrate thickness. All stresses are in the plane of the laminate. The stress in the surface of layer $2, \sigma_{2, \text { surface }}$, in layer 2 next to the interface to layer 1 , $\sigma_{2 \text {,interface }}$, and in layer 1 next to the interface to layer 2 , $\sigma_{1, \text { interface }}$, follow from the general relationships for the stress in multilayered composites ${ }^{3}$

$$
\sigma_{2, \text { surface }}=\frac{t_{1}\left(t_{1}^{3} E_{1}-3 t_{1} t_{2}^{2} E_{2}-2 t_{2}^{3} E_{2}\right)}{t_{2}\left(2 t_{1}^{3} E_{1}+3 t_{1}^{2} t_{2} E_{1}-t_{2}^{3} E_{2}\right)} \sigma_{1, \text { surface }},
$$

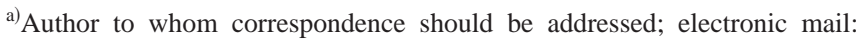
j.malzbender@fz-juelich.de
}

$$
\begin{gathered}
\sigma_{2, \text { interface }}=\frac{t_{1}\left(t_{1}^{3} E_{1}+3 t_{1} t_{2}^{2} E_{2}+4 t_{2}^{3} E_{2}\right)}{t_{2}\left(2 t_{1}^{3} E_{1}+3 t_{1}^{2} t_{2} E_{1}-t_{2}^{3} E_{2}\right)} \sigma_{1, \text { surface }}, \\
\sigma_{1, \text { interface }}=\frac{\left(4 t_{1}^{3} E_{1}+3 t_{1}^{2} t_{2} E_{1}+t_{2}^{3} E_{2}\right)}{\left(-2 t_{1}^{3} E_{1}-3 t_{1}^{2} t_{2} E_{1}+t_{2}^{3} E_{2}\right)} \sigma_{1, \text { surface }},
\end{gathered}
$$

where $t$ is the thickness and $E$ the elastic modulus (if the specimen is a plate $E /\left(1-\nu^{2}\right)$ has to be substituted). Since the stress can be a result of differences in thermal expansion but also intrinsic, growth related, the difference in strain is considered. If the strain is a result of thermal expansion $\epsilon$ $=\alpha \Delta T$ and $\Delta \epsilon=\Delta \alpha \Delta T$. Defining the strain in layer two as $\epsilon_{2}=\epsilon_{1}+\Delta \epsilon_{1,2}$ yields

$$
\begin{aligned}
\Delta \epsilon_{1,2}= & \frac{\left(t_{1}^{4} E_{1}^{2}+4 t_{1}^{3} t_{2} E_{1} E_{2}+6 t_{1}^{2} t_{2}^{2} E_{1} E_{2}+4 t_{1} t_{2}^{3} E_{1} E_{2}+t_{2}^{4} E_{2}^{2}\right)}{t_{2} E_{1} E_{2}\left(-2 t_{1}^{3} E_{1}-3 t_{1}^{2} t_{2} E_{1}+t_{2}^{3} E_{2}\right)} \\
& \times \sigma_{1, \text { surface }} .
\end{aligned}
$$

Here $E$ and $t$ are the average values during the expansion process. $^{3}$

These equations can be used to determine the stresses at various positions as exemplified for some literature data. Lead zirconate titanate (PZT) films are being used in electronic devices and microelectromechanical systems, where the reliability depends on the residual stresses in the film. ${ }^{5,6}$ A surface stress of $117 \mathrm{MPa}$ after cooling from $650{ }^{\circ} \mathrm{C}$ to room temperature has been determined using $\mathrm{x}$-ray diffraction for PZT films $(1 \mu \mathrm{m}$ thick, suffix 1) on Si substrates (525 $\mu$ m thick, suffix 2). ${ }^{5,6}$ A calculation of the stresses using the relationships presented above yields then $\sigma_{2 \text {,surface }}=0.4$ $\mathrm{MPa}, \sigma_{2 \text {,interface }}=-0.9 \mathrm{MPa}$ and $\sigma_{1 \text {,interface }}=117 \mathrm{MPa}$. The effective difference of the thermal expansion coefficients is $3.2 \mathrm{ppm}$. This effective value is a result of the difference in thermal expansion between paraelectric PZT and substrate above the Curie temperature $\left(\sim 300{ }^{\circ} \mathrm{C}^{6}\right)$ and between ferroelectric PZT and substrate below this temperature. Since the thermal expansion coefficient for $\mathrm{Si} \sim 5 \mathrm{ppm}$ and for paraelectric PZT $\sim 6.1 \mathrm{ppm},{ }^{6}$ an effective value of $\sim 11 \mathrm{ppm}$ results for the ferroelectric PZT. Further insight can be gained via a $\mathrm{x}$-ray diffraction measurement at elevated temperatures. 
Another example is the stresses in a solid oxide fuel cell. The surface stress in the center of a $30 \mu \mathrm{m}$ electrolyte (suffix 1, elastic modulus $207 \mathrm{GPa}$ ) on a $2 \mathrm{~mm}$ anode (suffix 2, elastic modulus $96 \mathrm{GPa}$ ) as determined using synchrotron radiation is $-590 /-690 \mathrm{MPa}$ (sintering temperatures $\left.1300 / 1500{ }^{\circ} \mathrm{C}\right){ }^{7} \quad$ This yields $\quad \sigma_{2 \text {,surface }}=-18 /-21 \mathrm{MPa}$, $\sigma_{2, \text { interface }}=36 / 42 \mathrm{MPa}$ and $\sigma_{1, \text { interface }}=-591 /-692 \mathrm{MPa}$. Although, in the case of a cell constrained against deflection the entire anode will be in a tensile stress state, the only tensile stress for the free cell exists in the anode at the interface with the electrolyte. Due to the brittle nature of the anodes used in solid oxide fuel cells, tensile stresses can be failure relevant depending on the modulus of rupture and the Weibull modulus of the material as well as the size of the cell in comparison with the fracture test specimen size. ${ }^{8,9}$ Hence, a cell sintered at higher temperatures will have a higher failure probability due to the higher tensile stress in the free anode surface. The effective difference of the thermal expansion coefficients is $2.53 / 2.55 \mathrm{ppm}$, hence relatively independent of the sintering temperature. The strain is a result of the difference in thermal expansion and intrinsic, sintering strains. The good agreement of the difference of the thermal expansion coefficients for the different sintering temperatures suggests that the sintering strain is only a minor contribution and that no relaxation effects occur between 1300 and $1500^{\circ} \mathrm{C}$.

As further example, a $5 \mu \mathrm{m} \mathrm{CeO}_{2}$ film on a $50 \mu \mathrm{m} \mathrm{Ni}$ substrate deposited at $600{ }^{\circ} \mathrm{C}$ resulted in an x-ray microdiffraction strain of $-0.2 \% .{ }^{10}$ Measurement from the Ni substrate showed no strain within the accuracy of the measurements $(\sim 0.02 \%)$. It was stated that this would be expected for a ductile, annealed metal substrate. ${ }^{10}$ However, the relationships given above permit one to assess the stress and strain in the Ni substrate in more detail. The stress $\sigma_{2, \text { surface }}=-14 \quad \mathrm{MPa}, \quad \sigma_{2 \text {,interface }}=28 \quad \mathrm{MPa} \quad$ and $\sigma_{1, \text { interface }}=-700 \mathrm{MPa}$. The effective difference in strain is $-0.21 \%$. The strain in the free surface of the Ni substrate is $-0.0065 \%$, hence below the limits of accuracy of the measurement, showing again the advantage of the methodology presented here. Note that, the theory assumes that all deformations are elastic, that all deformations are small compared to the layer thickness, and that the laminate is flat before elastic deformation. The sintering strain might be large, but does not contribute to the elastic deformation if the sintering stress is completely relaxed at high temperature.

Determining of the stresses in the surface of one layer for the bilayer system is followed by the determination of the stresses in one surface for the trilayer system.

The strain difference $\Delta \epsilon_{2,3}$ of the trilayer system is determined as $\Delta \epsilon_{1,2}$ for the bilayer system. If measurement performed in the surface of layer $1,{ }^{3} \sigma_{1 \text {,surface,trilayer }}=E_{1}\left(\epsilon_{0}\right.$ $\left.-\epsilon_{1}\right)$ has to be solved for $\Delta \epsilon_{1,2}$, if the stress is determined in the surface of layer $3,{ }^{3} \sigma_{3, \text { surface,trilayer }}=E_{3}\left[\left(t_{1}+t_{2}+t_{3}\right) \kappa\right.$ $\left.+\epsilon_{0}-\epsilon_{3}\right]$ has to be solved for $\Delta \epsilon_{1,2}$. The relationship for the strain $\epsilon_{0}$ and the curvature $\kappa$ are given in Ref. 3. The solution has to be inserted into the relevant equations for the stresses in surface and interface of layers 2 and 3.

As an example, a thermal barrier system, consisting of substrate, bond coat and thermal barrier coating, is considered. ${ }^{11}$ For the bilayer system the stress in the bond coat is measured as $-300 \mathrm{MPa}$ using $\mathrm{x}$-ray diffraction. The elastic modulus of substrate and bond coat are 214 and 170 $\mathrm{GPa}$, respectively, and thicknesses are $2 \mathrm{~mm}$ and $7 \mu \mathrm{m}$, respectively. ${ }^{11}$ Hence, $\sigma_{2 \text {,surface }}=-2 \mathrm{MPa}, \sigma_{2 \text {,interface }}=4 \mathrm{MPa}$, and $\sigma_{1, \text { interface }}=-300 \mathrm{MPa}$. The effective difference of the thermal expansion coefficients is $-2 \mathrm{ppm}$, the effective strain is $-0.18 \%$. After the deposition of the thermal barrier coating, the stress in the surface is $\sim-20 \mathrm{MPa}$ (thermal barrier coating as deposited, elastic modulus $70 \mathrm{GPa}$, thickness $300 \mu \mathrm{m}$, substrate $900 \mathrm{~K}$ furing deposition). ${ }^{11}$ The difference in strain determined above is $\Delta \epsilon_{2,3}=-0.18 \%$. Hence, $\sigma_{3, \text { surface }}=-6 \mathrm{MPa}, \sigma_{3, \text { surface }}=10 \mathrm{MPa}, \sigma_{2 \text {,surface }}=256$ $\mathrm{MPa}, \sigma_{2, \text { interface }}=256 \mathrm{MPa}$ and $\sigma_{1 \text {,interface }}=-21 \mathrm{MPa}$. The difference in strain $\Delta \epsilon_{2,3}=-0.15 \%$.

If a measurement of the stress in the surface of the bilayer composite is not possible a solution for the stresses and differences in strain can be obtained via a measurement of the stresses in the surface of layers 1 and 3. Then the relationships $^{3}$ for $\sigma_{1 \text {,surface,trilayer }}=E_{1}\left(\epsilon_{0}-\epsilon_{1}\right)$ and $\sigma_{3 \text {,surface,trilayer }}=E_{3}\left[\left(t_{1}+t_{2}+t_{3}\right) \kappa+\epsilon_{0}-\epsilon_{3}\right]$ have to be solved to yield $\Delta \epsilon_{1,2}$ and $\Delta \epsilon_{2,3}$.

The procedure is limited to trilayered composites if only the surface stresses can be determined. If a larger number of layers is to be analyzed the stress after each deposition step has to be measured and the strains/difference in thermal expansion can be determined as outlined above. Following the procedure, a determination of the stress profile and thermal expansion coefficients for composites of a number of alternating bilayers or trilayers is also possible. In this case the measurement of the stress in the surface has obvious advantages over a determination of the stresses from the curvature since, although the stresses increase, the curvature decreases with the number of alternating layers and the neutral axis approaches the middle of the specimen. The stress in the free surface of layer 1 approaches asymptotically ${ }^{3} \sigma_{1 \text {,surface, } n \text {-layer }}$ $=E_{1}\left(\epsilon_{0}-\epsilon_{1}\right)$ and in the surface of layer $n,{ }^{3} \sigma_{n \text {, surface, } n \text {-layer }}$ $=E_{n}\left(\epsilon_{0}-\epsilon_{n}\right)$, hence, if only two different materials are present a measurement of only one surface stress permits a determination of the misfit strain or difference in thermal expansion coefficient.

The work was financially supported by the European Union within the project "Component Reliability in Solid Oxide Fuel Cell Systems for Commercial Operation (CORESOFC)". The scientific support of R. W. Steinbrech and L. Singheiser is kindly acknowledged.

${ }^{1}$ R. J. Roark and W. C. Young, Formulas for stress and strain (McGrawHill, Singapore, 1984).

${ }^{2}$ J. Malzbender and R. W. Steinbrech, J. Mater. Res. 18, 1975 (2003).

${ }^{3}$ J. Malzbender, J. Appl. Phys. 95, 1780 (2004).

${ }^{4}$ B. B. He, Powder Diffr. 18, 71 (2003).

${ }^{5}$ W.-H. Xu, D. Lu, and T.-Y. Zhang, Appl. Phys. Lett. 79, 4112 (2001).

${ }^{6}$ M.-H. Zhao, R. Fu, D. Lu, and T.-Y. Zhang, Acta Mater. 50, 4241 (2002).

${ }^{7}$ H. Yakabe, Y. Baba, Y. Matsuzaki, and Y. Yoda, J. Soc. Mater. Sci. Jpn. 52, 89 (2003).

${ }^{8}$ J. Malzbender, R. W. Steinbrech, and L. Singheiser, Proceedings of the Fifth European SOFC Forum, edited by J. Huijsmans, Luzern, 2002, Vol. 2, p. 1091.

${ }^{9}$ J. Malzbender and R. W. Steinbrech, Surf. Coat. Technol. 176, 165 (2004).

${ }^{10}$ J. D. Budai, W. Yang, N. Tamura, J.-S. Chung, J. Z. Tischler, B. C. Larson, G. E. Ice, C. Park, and D. P. Norton, Nat. Mater. 2, 487 (2003).

${ }^{11}$ V. Teixeira, M. Andritschky, W. Fischer, H. P. Buchkremer, and D. Stöver, Surf. Coat. Technol. 120,121, 103 (1999). 